\title{
Avaliação do tratamento farmacoterapêutico em jovens com transtorno de ansiedade durante a pandemia
}

Evaluation of pharmacotherapeutic treatment in young people with anxiety disorder during the pandemic

Evaluación del tratamiento farmacoterapéutico en jóvenes con trastorno de ansiedad durante la pandemia

\author{
Yana Letícia de Sousa Gomes \\ ORCID: https://orcid.org/0000-0002-2144-5573 \\ Centro Universitário Santo Agostinho, Brasil \\ E-mail: legomess50@gmail.com \\ Caio Lima da Silva Pinto \\ ORCID: https://orcid.org/0000-0002-7774-111X \\ Centro Universitário Santo Agostinho, Brasil \\ E-mail: caiolimasilva93@gmail.com \\ Raimundo Nonato Cardoso Miranda Júnior \\ ORCID: https://orcid.org/0000-0003-2937-6143 \\ Centro Universitário Santo Agostinho, Brasil \\ E-mail: jrfarmaceutico@ hotmail.com
}

\begin{abstract}
Resumo
O Transtorno de ansiedade tem afetado os jovens com o isolamento social anulando assim a vida social das pessoas decorrendo assim o aumento das crises de ansiedade. Durante as epidemias, o número de pessoas cuja a saúde mental é afetada tende a ser maior que o número de pessoas com infecções (ORNELL et al. 2020). Segundo o Ministério da Saúde do Brasil (2020) um evento como esse ocasiona perturbações psicológicas e sociais que afetam a capacidade de enfrentamento de toda a sociedade, em variados níveis de intensidade e propagação. O objetivo foi entender como os jovens estão comportando-se com transtorno de ansiedade diante a pandemia. Este estudo referiu-se a uma revisão de literatura integrativa do tipo explicativa quantitativa. Esperou-se entender como os jovens estão comportando-se com o transtorno de ansiedade durante a pandemia SARS-CoV-2 bem como responder quais a consequências que a doença tem trago e se houve ocorrência dos aumentos de casos, tendo vista que os jovens tenham consciência da melhoria da qualidade de vida que o tratamento farmacoterapeutico possa lhe proporcionar.
\end{abstract}

Palavras-chave: Pandemia; Transtorno de ansiedade; Tratamento farmacoterapeutico.

\begin{abstract}
Anxiety Disorder has affected young people with social isolation, thus canceling out people's social lives, leading to an increase in anxiety crises. During epidemics, the number of people whose mental health is affected tends to be greater than the number of people with infections (ORNELL et al. 2020). According to the Ministry of Health of Brazil (2020), an event like this causes psychological and social disturbances that affect the coping capacity of the entire society, at varying levels of intensity and spread. The objective was to understand how young people are behaving with anxiety disorder in the face of the pandemic. This study referred to an integrative literature review of the quantitative explanatory type. It was hoped to understand how young people are behaving with anxiety disorder during the SARS-CoV-2 pandemic, as well as to answer what consequences the disease has brought and if there was an increase in cases, considering that young people have awareness of the improvement in the quality of life that pharmacotherapeutic treatment can provide.
\end{abstract}

Keywords: Pandemic; Anxiety disorder; Pharmacotherapeutic treatment.

\section{Resumen}

El trastorno de ansiedad ha afectado a jóvenes con aislamiento social, afectando así la vida social de las personas, provocando un aumento de los ataques de ansiedad. Según el Ministerio de Salud de Brasil (2020). Un evento como este provoca perturbaciones psicológicas y sociales que afectan la capacidad de afrontamiento de toda la sociedad, en distintos niveles de intensidad y extensión, con el objetivo de comprender cómo se están comportando los jóvenes con trastorno de ansiedad generalizada durante la pandemia. Metodología Se trata de un estudio transversal apoyado en un 
enfoque cuantitativo y cualitativo. Se espera comprender cómo se están comportando los jóvenes con trastorno de ansiedad durante la pandemia del SARS-CoV-2, así como responder qué consecuencias ha traído la enfermedad y si hubo un aumento de casos, dado que los jóvenes las personas son conscientes de la mejora en la calidad de vida que puede proporcionar el tratamiento farmacoterapéutico.

Palabras clave: Pandemia; Trastorno de ansiedad; Tratamiento farmacoterapéutico.

\section{Introdução}

A COVID-19, ocasionada pelo novo corona vírus, foi inicialmente detectada em 2019 na cidade de Wuhan, capital da província da China Central. Ela atingiu as pessoas em diferentes níveis de complexidade, sendo os pacientes mais graves acometidos de: formação de trombos, quadro inflamatório agudo e insuficiência respiratória aguda que requer cuidados hospitalares intensivos, incluindo a ventilação mecânica. (Centers For Disease Control 2020).

Esta recente pandemia, gerada pelo vírus SAR-COV-2 (LIANG, 2020) representa um grande desafio para a sociedade, pois trata-se de um evento potencialmente estressante, tendo em vista as medidas necessárias para a prevenção e contenção da doença, como: distanciamento social, aumento de internações, uso de equipamento de proteção individual (EPI) e cuidado com a higiene pessoal. A isso soma-se os impactos econômicos, com paralisação das empresas, escolas, órgãos públicos e retardar o crescimento econômico; impactos políticos, devido ao endividamento público e sociais com enorme crescimento do desemprego, aumento da informalidade e avanço da pobreza. (Van Bavel et al ,2020).

Durante as epidemias, o número de pessoas cuja a saúde mental é afetada tende a ser maior que o número de pessoas com infecções (Ornell et al. 2020), sendo dessa forma prejudicial ao convívio social e profissional dos jovens, aumentando a insegurança em relação aos seus possíveis futuros consequentemente causando frustações e mudanças de humor (Athanasio, 2020).

Books SK, et al. (2020) enfatiza que a quarentena está frequentemente associada com um efeito psicológico negativo. O estudo de Itu D, et al. (2020) corrobora com esta premissa quando revela que mais de $80 \%$ dos participantes da pesquisa estavam preocupados com a pandemia da COVID-19 e aproximadamente $40 \%$ dos participantes estavam paranoicos com o pensamento de contrato a nova doença.

Segundo o Ministério da Saúde do Brasil (2020), um evento como esse ocasiona perturbações psicológicas e sociais que afetam a capacidade de enfrentamento de toda a sociedade, em variados níveis de intensidade e propagação. O Brasil é, atualmente, o país que possui em sua população uma prevalência de 9,3\% de indivíduos diagnosticado com transtorno de ansiedade, sendo o maior país do mundo com tal distúrbio em evidencia (World Health, 2017). Por isso, este trabalho visa entender como estão comportando os jovens com transtorno de ansiedade durante a pandemia do SARS-CoV-2, e os principais tratamentos farmacológicos e não farmacológicos para ansiedade.

\section{Metodologia}

O presente estudo realizou por meio de uma revisão de literatura integrativa do tipo explicativa quantitativa, com intuito de responder o seguinte Problema de Pesquisa: "Como o transtorno de ansiedade está afetando os jovens durante a pandemia?".

O levantamento de informações foi realizado entre o período de agosto de 2020 até junho de 2021, nas bases de dados da Plataforma da Biblioteca Virtual em Saúde (BVS) utilizando artigos nas bases de dados, Scientific Eletronic Library Online (SCIELO), Literatuta Latino-Americana e do Caribe em Ciências da Saúde (LILACS), Medical Literature Analysis and Retrieval System Online (MEDLINE). Foram utilizados os descritores retirados: Pandemia; Transtorno de ansiedade; Tratamento farmacoterapêutico.

Após uma revisão sistematizada dos dados por meio da leitura minuciosa dos artigos, foram selecionados 60 artigos no 
total, sendo que 42 foram incluídos, pois além de serem dos últimos 10 anos os mesmos possuem relação com o objetivo do estudo que é o comportamento dos jovens com transtorno de ansiedade durante a pandemia do SARS-CoV-2 e os principais tratamentos farmacoterapêuticos para o mesmo, e foram excluidos 18artigos por serem antigos, ou seja, não estavam dentro dos ultimos 10 anos ou não tratavam do tema em questão.

Por não se tratar de pesquisa com humanos e sim de uma pesquisa literária, não houve necessidade de submeter ao CEP.

\section{Resultados e Discussão}

Após uma revisão sistematizada dos dados por meio da leitura minuciosa dos artigos, além da aplicação dos critérios de inclusão e exclusão descritos na metodologia foram selecionados aqueles artigos que pudessem guardar relação com o objetivo do estudo, respondendo a questão norteadora desta pesquisa.

Os resultados foram submetidos a uma tabulação, Tabela 1 e Tabela 2, onde os dados foram sintetizados e dispostos de forma sistemática para melhor exposição das informações, em que na tabela 1 trata-se dos principais medicamentos usados no tratamento da ansiedade, além de demostrar, também, alguns dos tratamentos não farmacológicos. Já na tabela 2 fez-se uma comparação das buscas dos principais medicamentos para a ansiedade, entre o ano de 2020 e 2021.

De acordo com a Associação Americana de Psiquiatria (APA) a ansiedade é considerada um dos transtornos mentais que mais acometem a população jovem e adulta. (APA, 2017; Brasil, 2015). Segundo Vasconcellos (2020), a ansiedade tem cinco fases de zero a quatro) e normalmente, o tratamento medicamentoso passa a ser recomendado na fase dois, quando o distúrbio traz, por exemplo, prejuízos no trabalho, com baixa no rendimento, e na vida socioafetiva. Na fase um, quando se tem mal-estar e sintomas leves, a pessoa vai tomar a medicação de um a três meses, ainda assim a psicoterapia pode ser tão ou mais eficiente que os remédios, principalmente se combinada com hábitos saudáveis e terapias complementares.

No nível 3 em seguinte, quando o paciente já tem prejuízos na vida, o medicamento vai ser companheiro por um ano. Nas fases seguintes, a medicação é revisada de ano em ano e, caso haja melhoras, é retirada de forma gradual.

Tabela 1 - Principais Tratamentos Farmacológicos e Não Farmacológicos para ansiedade.

\begin{tabular}{|c|c|}
\hline Tratamentos Farmacológicos & Tratamentos Não Farmacológicos \\
\hline Inibidores Seletivos da Recaptacão de Serotonina & Psicoterapia: \\
\hline (ISRS): & Terapia Cognitivo-comportamental \\
\hline $\begin{array}{l}\text { Cloridrato de fluoxetina; sertralina; paroxetina; } \\
\text { Escitalopram }\end{array}$ & Medicina Integrativa: \\
\hline Tricíclicos: & $\begin{array}{l}\text { Exercícios de respiração diafragmática; meditação; } \\
\text { acumputura, musicoterapia; massagens; }\end{array}$ \\
\hline Blprazolam; clonazepam, zolpidem & $\begin{array}{l}\text { Tratamentos Naturais: } \\
\text { Fitoterápicos }\end{array}$ \\
\hline $\begin{array}{l}\text { ISRSN: } \\
\text { Venlafaxina }\end{array}$ & \\
\hline $\begin{array}{l}\text { B-bloqueadores } \\
\text { Propranolol; atenolol }\end{array}$ & \\
\hline
\end{tabular}

Fonte: Dados da pesquisa (2021).

As diretrizes estabelecidas nas terapias farmacológicas para o tratamento da Ansiedade podem ser resumidas nos 
psicorfarmárcos que são consideradas de primeira linha no tratamento farmacológico na ansiedade, tanto pelo fato de terem se mostrado eficazes quanto pela segurança de seus efeitos adversos. Essas drogas de escolha são inibidores seletivos de receptação de serotonina (ISRS) que agem inibindo a recaptação da serotonina na fenda sináptica aumentando assim os níveis de neurotransmissores para execer atividades serotoninergica, e os inibidores seletivos de receptação de serotonina e noradrenalina (IRSN). Apesar dos benzodiazepínicos (BZD) também apresentarem forte evidência de eficácia, não são considerados medicações de primeira linha em função do perfil pouco favorável de efeitos adversos e risco de abuso e dependência (Levitan, 2011).

Os betabloqueadores embora utilizados para ansiedade a recomendação do seu uso limitam-se em certas situações que não fazem parte do índividuo, visando reduzir os sitomas somados da ansiedade como as palpitações cardíacas (diminuindo a taquicardia), respiração rápida e sudorese (Coelho, et al 2017).

As terapias complementares são estratégias não farmacológicas, de cunho científico ou não, que têm sido praticadas por algumas pessoas e tidas como soluções amenas para diversas enfermidades, dentre elas, a ansiedade. Dentre essas estratégias, pode-se citar a dança, a pintura, a meditação, as técnicas de relaxamento, a massagem, dentre outros (Braz et al., 2011).

Para Barros e Gomes (2019), a atividade física mostra-se um método não farmacológico de terapia para depressão e ansiedade, pois durante o exercício ocorre a liberação de várias substâncias na corrente sanguínea que aumentam o bem estar e prazer, mas relata também que por conta do período de isolamento na pandemia também ocorreu o aumento da inatividade física. As pessoas estão passando a maior parte do tempo sentadas por causa do trabalho (home-office), estudo, leitura, TV, celular (jogos e/ou redes sociais).

As terapias da fala (psicoterapia), em geral, ajudam os pacientes a identificar os fatores que deflagram a ansiedade, como falsas crenças, e a encontrar formas mais saudáveis de lidar com os sintomas e as dificuldades que o transtorno cria. A psicanálise e as terapias psicodinâmicas podem ser úteis nesse aspecto, mas a TCC (terapia cognitivo-comportamental), que tem um caráter mais prático, é a que tem demonstrado maior eficácia segundo evidências científicas (Oliveira, 2019; Beck,2013; Clark, Beck 2012).

Exercícios de respiração diafragmática, como os da ioga, também são considerados úteis. Além disso, técnicas de relaxamento, neurofeedback, biofeedback, alongamentos, massagens, musicoterapia, acupuntura, banhos quentes e ervas como camomila, passiflora e valeriana pode ajudar no controle dos sintomas e aumentar o sucesso dos tratamento convencionais (Vasconcellos, 2020).

Um levantamento inédito do Consulta Remédios(2021), vide tabela 2, comprovou que houve um aumento de até 113\% na procura de medicamentos destinados ao tratamento de insônia, ansiedade e depressão, comparando os seis meses anteriores à pandemia - agosto de 2019 a fevereiro de 2020 - com o respectivo período recente, agosto de 2020 a fevereiro de 2021. Hemitartarato de Zolpidem - destinado ao tratamento da insônia - lidera a lista e teve o aumento mais expressivo de buscas no mesmo comparativo: 865.985 buscas entre 08/20 e 02/21, uma alta de $113 \%$ na comparação com o mesmo período do ano anterior (NUNES, 2021). 
Tabela 2 - Buscas comparativas antes e durante a pandemia no período de - 08/20 a 02/21 em relação ao ano anterior (08/19 a 02/20).

\begin{tabular}{lccc}
\hline Medicamento & $\mathbf{0 8 / 1 9}$ a 02/20 & $\mathbf{0 8 / 2 0}$ a 02/21 & Crescimento \\
\hline $\begin{array}{l}\text { Hemitartarato de } \\
\text { Zolpidem }\end{array}$ & 405.374 & 865.985 & 113,63 \\
\hline $\begin{array}{l}\text { Cloridrato de } \\
\text { Fluoxetina }\end{array}$ & 443.831 & 806.625 & 81,74 \\
\hline Oxalato de & & & \\
Escitalopram & 552.590 & 714.727 & 29,34 \\
\hline Sertralina & & & 55,35 \\
\hline clonaepam & 416.924 & 647.699 & 83,43 \\
\hline
\end{tabular}

Fonte: Dados do Consulta Remédios (2021).

O Cloridrato de Fluoxetina, indicado para depressão, vem na sequência. O medicamento foi procurado 806.625 vezes no período de agosto/2020 a fevereiro/2021. Um aumento de 81,74\% em relação ao mesmo período anterior. Oxalato de Escitalopram - comumente destinado ao tratamento de depressão - que apresentou um crescimento de buscas de $29,34 \%$, totalizando 714 mil buscas; a Sertralina, também com a mesma finalidade, que registrou um aumento de procura de 55,35\%, com 1,28 milhão de buscas; e, por fim, o Clonazepam, receitado para transtornos psicológicos, cujo expressivo aumento foi de $83,43 \%$, um total de mais 495 mil buscas (Medicina S/A, 2021).

Antes da pandemia já existia tratamento não farmacológico para ajudar pessoas com crises de ansiedade, que são métodos de relaxamento diários para reduzir os sintomas físicos de tensão. Recomenda-se associar medicação, terapia e mudanças no estilo de vida, como iniciar atiividades físcas (Vasconcellos, 2020).

\section{Considerações Finais}

A pandemia associou-se à um aumento no aparecimento de transtornos mentais, assim como a ansiedade sendo um fator preditor para um elevado consume de psicofármacos e buscas de formas alternativas além do medicamentoso.

Desta forma, tornar-se necessário o desenvolvimento de políticas de alerta frente os riscos associados ao aumento de uso de tais fármacos, com a adoção de ações de promoção visando o uso racional dos mesmos.

Esse artigo possibilitou conhecer de forma mais aprofundada a avaliação do tratamento farmacoterapêutico em jovens com transtorno de ansiedade durante a pandemia, em que pesquisas demonstraram que nesse período dificil seja vivida uma elevada carga de momentos, experiências e emoções negativas, e por conta disso, a grande necessidade de ter cuidado com o psicológico.

\section{Referências}

APA-American Psychiatric Association. (2014). Manual Diagnóstico e Estatístico de Transtornos Mentais, DSM-5. Artmed.

American Psychiatric Association. Anxiety Disorders. APA. https://www.psychiatry.org/patients-families/anxietydisorders/what-are-anxiety-disorders.

Andreatini R, Boerngen-Lacerda R, \& Zorzetto Filho D. (2001) Pharmacological treatment of generalized anxiety disorder: future perspectives. Rev Bras Psiquiatr. 23(4):233-42

Athanasio, B. S., et al. (2020) Saúde mental de crianças e adolescentes na pandemia da covid-19. Livro de resumos da xvii jornada acadêmica de saúde mental: Faculdade De Medicina Da Universidade Federal De Minas Gerais.

American Psychiatry Association. (2014). Diagnostic and Statistical Manual of Mental disorders-DSM-5. (5th.ed.), American Psychiatric Association. Http://Pepsic.Bvsalud.Org/Scielo.Php?Script=Sci_Nlinks\&Ref=3209145\&Pid=S1808-5687201700010000800001\&Lng=Pt. 
Associação Brasileira De Psiquiatria. (2008) Projeto Diretrizes.

Bajardi, P., Poletto, C., Ramasco, J.J., Tizzoni, M., Colizza, V., \& Vespignani, A. (2011). Humanmobility networks, travelrestrictions, andthe global spread of 2009 H1N1 Pandemic. PlosOne, 6(1), e16591. http://doi.org/10.1371/journal.pone.0016591

Beck, J. S. Terapia cognitivo-comportamental: teoria e prática. (2a ed). Artmed Editora. (2013).

Barros, R.C.S., \& Gomes R.L.R. "O exercício físico como ferramenta de motivação e produtividade no meio corporativo”, Revista Observatorio de la Economía Latinoamericana. 2019.

Brasil. Secretaria da Saúde de Curitiba. Biblioteca Virtual em Saúde. Ansiedade. Ministério da Saúde, https://bvsms.saude.gov.br/dicas-em-saude/470-ansiedade.

Brooks, S. K., Webster, R. K., Smith, L. E., Woodland, L., Wessely, S., Greenberg, N., \& Rubin, G. J. (2020). The psychologicalimpactofquarantineandhowtoreduce it: Rapidreviewoftheevidence. The Lancet, 395(102227), 912-20. http://doi.org/10.1016/S01406736(20)30460-8.

Centers for Disease Control Prevention [CDC] (2020b). SevereoutcomesamongpatientswithCoronavirusDisease 2019 (COVID-19) - United States, February 12March 16, 2020. MorbidityandMortalityWeeklyReport, 69(12), 343-346. http://doi.org/10.15585/mmwr.mm6912e2

Clark, D., \& Beck, A. Terapia cognitiva para os transtornos de ansiedade. Artmed, 2012. p. 389-445.

Classificação de Transtornos Mentais e de Comportamento da CID-10: Descrições Clínicas e Diretrizes Diagnósticas. Trad. Dorgival Caetano. Porto Alegre: Artes Médicas, 1993.

Coedeiro, C. H. G., Chung, M. C., \& Sacramento, L. V. S. interações medicamentosas de fitoterápicos e fármacos: hypericum perforatum e piper methysticum. Revista brasileira de farmacognosia.2005.15(3):272-278.

Danese, A., \& Smith, P. (2020). Debate: Recognisingandrespondingtothe mental healthneedsofyoungpeople in the era of COVID-19. Child and adolescent mental health, 25(3), 169-170. https://doi.org/10.1111/camh.12414.

Faustino, T. T., Almeida, R. B. de, \& Andreatini, R. (2010) Plantas medicinais no tratamento do transtorno de ansiedade generalizada: uma revisão dos estudos clínicos controlados. Revista Brasileira de Psiquiatria, 32(4), 429-436.

Figueiredo, C. S., et al. COVID-19 pandemic impact on children andadolescents' mental health: Biological, environmental, and social factors. Progress in neuropsycho pharmacology \& biological psychiatry, 106, 110171. https://doi.org/10.1016/j.pnpbp.2020.110171.

Guessoum, S. B., et al. Adolescent psychiatric disorders during the COVID19 pandemic and lockdown. Psychiatry research, 291 , 113264. https://doi.org/10.1016/j.psychres.2020.113264

Hartmann, P. B., O uso abusivo de psicofármacos nos EUA entre adolescentes e jovens. PEBMED, https://pebmed.com.br/o-uso-abusivo-de-psicofarmacos-noseua-entre-adolescentes-e-jovens/.

Knapp, P. Princípios da Terapia Cognitiva. In: Knapp, P.(org.) Terapia cognitivo-comportamental na prática psiquiátrica. Artes Médicas, 2004

Leahy, R. L., Tirch, D., \& Napolitano, L. A. Regulação emocional em psicoterapia: um guia para o terapeuta cognitivo-comportamental. Porto Alegre: Artmed, 2013.

Levitan Michele, Et al. Diretrizes da Associação Médica Brasileira para o tratamento do transtorno de ansiedade social. Revista Brasileira de Psiquiatria $\bullet$ vol $33 \cdot n^{\circ} 3 \cdot$ set., 2011 .

Liang, T. Zhejiang University School of Medicine. Handbookof COVID-19: Preventionandtreatment. Paris: InternationalAssociationofUniversities, UNESCO. http://www.zju.edu.cn/english/2020/0323/c19573a1987520/page.htm.

Lopes, K. C. S. P., \& Santos, W. L. (2018) Transtorno de ansiedade. RevInicCient Ext. 1(1): 45-50. Liang, T. (2020). ZhejiangUniversitySchoolof Medicine. Handbookof COVID-19: Preventionandtreatment. Paris: InternationalAssociationofUniversities, UNESCO.

Marques, E. L. L., \& Borba, S. de. (2016) Como lidar com o transtorno de ansiedade generalizada na perspectiva da terapia cognitivo-comportamental. SynThesis Revista Digital FAPAM, 7(7), 82-97.

Menezes, A. K. da S., Moura, L. F. de, \& Mafra, V. R. (2017) Transtorno de ansiedade generalizada: uma revisão da literatura e dados epidemiológicos. 10.18606/2318-1419/amazonia.sci.health.v5n3p42-49Revista Amazônia Science \& Health.

Mary, A. et al. (2017) An overview on anxiety: etiology and therapy. Journal of Pharmaceutical Research, 16(1), 32-37.

Medicina S/A. Busca por ansiolíticos e antidepressivos cresce mais de 100\% na pandemia. 2021. https://medicinasa.com.br/busca-ansioliticosantidepressivos/.

Miliauskas, C. R., \& Faus, D. P., (2020) Saúde mental de adolescentes em tempos de Covid-19: desafios e possibilidades de enfrentamento. Revista de Saúde Coletiva, 30(4), e300402.

Oliveira, A. C. (2019) Eficácia da terapia cognitivo comportamental no tratamento da depressão: revisão integrativa. Rev. Bras. Ter. Cogn. 15(1):29-37.

Ornell, F. et al. (2020) Pandemia de medo e COVID-19: impacto na saúde mental e possíveis estratégias. vista debates in psychiatry - Aheadofprint

Pagani, C. A., \& Silva, B. F. Uso popular de plantas medicinais no tratamento da ansiedade. Universidade Do Planalto Catarinense - UNIPLAC. 2016. 
Research, Society and Development, v. 10, n. 15, e404101522958, 2021

(CC BY 4.0) | ISSN 2525-3409 | DOI: http://dx.doi.org/10.33448/rsd-v10i15.22958

http://www.uniedu.sed.sc.gov.br/wp-content/uploads/2017/09/Claudia-Arruda-Pagani.pdf.

Rego, o. K., \& Maia, J. L. F. Ansiedade em adolescentes no contexto da pandemia por COVID-19. Research, Society and Development, v. 10, n. 6, e39010615930, 2021

Sadock, B. J., \& Sadock, V. A. (2020) Kaplan \& Sadock’sSynopsisofPsychiatry, (10th ed.).

Sadock, B. J., \& Sadock, V. A. (2020) Compêndio de psiquiatria: ciência do comportamento e psiquiatria clínica. (9a ed.), Artmed.

Sadock, B. J., Sadock, V. A., \& Ruiz, P. (2017) Compêndio de Psiquiatria: ciência do comportamento e psiquiatria clínica. (11a ed.), Artmed.

Sousa, M. S. P., et al. Uso de antidepressivos e ansiolíticos entre estudantes do curso de farmácia em uma instituição privada e uma pública do interior da Bahia. Research, Society and Development, 10(8), e29610817177. 2021.

Van Bavel, J. J., Baicker, K., Boggio, P., Capraro, V., Cichocka, A., \& Willer, R. (2020). Using social andbehavioralsciencetosupport COVID-19 pandemic response. PsyArXiv, 1-50.

Vasconcellos, H., É possível combater a ansiedade sem remédio? https://www.uol.com. br/vvabem/noticias/redacao/2020/10/23/e-possivel-tratar-a-ansiedadesemremedios.htm?ne $\mathrm{xt}=0001 \mathrm{H} 221 \mathrm{U} 11 \mathrm{~N} \& \mathrm{cmpid}$.

World Health Organization. (2017). Depression and other common mental disorders: global health estimates (No. WHO/MSD/MER/2017.2). World Health Organization.

World Health Organization [WHO]. (2020a). Coronavirusdisease 2019 (COVID-19) SituationReport - 78. 